\title{
PHYSICS FORUM
}

\section{THE PHYSICS OF SOLIDS}

\section{A. DuBridge}

\author{
University of Rochester, Rochester, New York
}

I HAVE been asked to review before this group some of the developments in physics during the past twenty-five years, particularly as they relate to our understanding of the physics of solids. I am going to address my remarks particularly to those who have not been in a position to follow these developments but who are interested in getting a general idea of the new ideas which have proved of value in understanding and correlating the properties of solids.

I think it is of interest to imagine oneself translated back twenty-five years to the year 1912. This was a rather "normal" year in the development of physics. So far as I can recall it was not marked by any particularly startling developments but was a year of sound progress. $\mathrm{X}$-rays, radioactivity, and the electron had been discovered nearly fifteen years previously and had stimulated an enormous amount of research. The quantum theory had been proposed and had met with some success but was not yet taken too seriously by most physicists. The theory of relativity was being discussed and had proved its value in astronomical problems but its use in everyday physics seemed remote. But these developments had opened the way for a fundamental attack on the problem of atomic structure and in 1912, in Rutherford's laboratory, Geiger and Marsden were completing their experiments on the scattering of alpha-particles. These experiments laid the basis for our present picture of the nuclear atom consisting of a massive positively charged nucleus surrounded by a swarm of planetary electrons.

* From an address given before the meeting of the physicists of Upper New York State at Ithaca, N. Y., November 6, 1937.
In the following year Niels Bohr, combining Rutherford's nuclear atom with Planck's quantum postulate, proposed his famous theory of atomic structure. Sommerfeld quickly showed that this theory could be extended through the application of the theory of relativity. Thus in one stroke physicists acquired a new and surprising picture of atomic structure which correlated a vast amount of material which had been brought to light in the previous twenty years and which proved an enormous stimulus to further experimental and theoretical work. In spite of the interruption caused by the war, the next ten years, 1915-1925, were exceedingly productive ones and by 1922 the general predictions of Bohr's theory had been fully confirmed experimentally. Studies in the field of spectroscopy established with uncanny precision Bohr's fundamental idea that the electrons within an atom could occupy only certain discrete energy levels. This idea remains today the outstanding fundamental principle of atomic theory.

However, there were logical difficulties in Bohr's simple picture and although it was elaborately extended and on the whole met with astounding success, there were certain features which failed to agree with experiment. As experimental technique developed and became more precise these failures of the theory became more pronounced. The difficulties involved predictions concerning the structure of the spectrum of the radiation which atoms emitted. But there were also questions about the nature of the radiation itself. The quantum theory of radiation had proved surprisingly successful and the discovery of the Compton effect seemed to remove the last doubt of its correctness. But it shared honors 
with an equally successful wave theory of radiation, whose fundamental postulates were apparently in conflict with the quantum theory.

In 1926 the situation was clarified. In that year Schroedinger developed the suggestion, previously made by de Broglie, that the motion of electrons within an atom could be treated as a wave motion. This idea led to a logical explanation of the existence of discrete energy levels within the atom. These discrete orbits were now visualized as standing wave patterns. In the hands of Schroedinger, Heisenburg, and Dirac a new theory of quantum mechanics grew up which retained the successful features of Bohr's theory but removed its difficulties and removed the discrepancies with experiment. Quantum and wave were no longer conflicting ideas but merely two ways of describing the same thing. This idea that matter also posséssed a wave aspect quickly found justification in the experiments of Davisson and Thompson on the diffraction of electrons.

By 1932 quantum mechanics had been generalized, extended and applied to a wide range of phenomena with marked success. Physicists had become used to the strange mathematical garb in which the theory was dressed and the new styles now seemed not only logical but satisfying and even attractive.

Since 1932 new difficulties have arisen. Experimental technique has developed to the point where physicists are no longer content to study. the behavior of the external electrons in an atom whose behavior could be so accurately predicted by quantum mechanics. Attention is now directed at the nucleus where distances are small compared to the size of the particles and where new types of force fields come into play. Some of the new discoveries in nuclear physics have been adequately taken into account by extensions of quantum mechanics. But there still exist many serious difficulties. The theory of the nucleus is not at all fully understood and we may be on the verge of totally new developments in this field.

However, we are not today concerned with nuclear physics, fascinating a field though it may be. We now turn to the question of whether the quantum theory of the structure and behavior of individual atoms and electrons can be applied to problems which the engineer would regard as more "practical," that is, to problems having to do with objects which we can feel and handle. In other words, does quantum theory have any application to problems of industrial importance?

Emphatically it does. Everyone must admit that solid bodies are of very great practical importance. We use them to build motors and bridges, automobiles and optical instruments. We make photographic plates, electric lights, photoelectric cells and television tubes. In fact nearly every product of our industrial system depends upon the mechanical, thermal, electrical and optical properties of solids. Now quantum theory has pointed the way to a fuller understanding of just these things. It has supplied the basis for the theoretical treatment of some of the properties of solids which only a few years ago could not be understood at all. It has started a revolution in a field which was until recently a no-man's land, a land left to a host of empirical relationships with but little underlying correlating theory.

The modern quantum theory of solids in its entirety is a complex mathematical structure but some of the fundamental ideas involved are essentially simple. We have already mentioned the fact that one of the fundamental properties of an atom is the system of discrete energy levels in which the electrons are confined. There is a further restriction on the way in which these levels may be occupied: any given energy level or "quantum state" can never be occupied simultaneously by more than two electrons and these two must have their spins oppositely directed (Pauli principle). In a normal atom containing many electrons the lower quantum states will be fully occupied each by two electrons. The outer states may be empty or only partially filled. When the atom is disturbed in certain ways some of the outer electrons may move further out and occupy temporarily these unfilled levels. When these electrons drop back to their normal position the atom emits radiation of a wave-length determined by the energy change of a falling electron.

When two or more similar atoms combine to form a molecule the energy level scheme is disturbed. For the low lying levels (the orbits closest to the nucleus) the change is very slight but the pattern of levels available to the outer or 
valence electrons is quite different. It is no longer possible to say that a particular valence electron belongs to one atom or another. It belongs to the molecule as a whole and occupies levels which are characteristic of the whole molecule. There is a correlation, however, between the molecular energy levels and those of the individual atoms. A given atomic quantum state may be split in two and its energy value shifted when the molecule is formed. Quantum mechanics provides the method for computing the new energy level scheme.

Now the central idea of the new quantum theory of solids is simply that a crystalline solid body may be regarded as a single large molecule. For a crystal of ordinary size this "molecule" will contain in the neighborhood of $10^{23}$ atoms. When these atoms are crowded together in a regular array the energy level scheme for the electrons is again altered. The alteration is slight for the deep lying $x$-ray levels; larger for the outer or valence levels. The new level scheme, however, is still correlated with the levels of the individual atoms and quantum mechanics provides again the method for calculating the new pattern. The valence electrons of the atoms no longer belong to individual atoms but to the crystal molecule as a whole. In this sense the valence electrons are "free" electrons. The crystal will have a characteristic energy level scheme which depends upon the nature of the atoms of which it is composed and on their crystal arrangement. The number of individual energy levels will of course be very large. They must accommodate $10^{23}$ electrons ( 1 to 4 for each atom) with still no more than two electrons in each level.

The shift of the energy level diagram in going from atom to molecule to crystal is crudely represented by the diagram of Fig. 1. In general each group of atomic levels will be broadened out into a band or zone of crystal levels, each zone containing $N$ closely spaced levels where $N$ is the number of atoms in the whole crystal. In some cases there will be wide gaps in energy between the successive bands. These gaps represent energy values which are forbidden to the electron. Sometimes these gaps will be small and sometimes the bands will overlap leaving no gap at all.

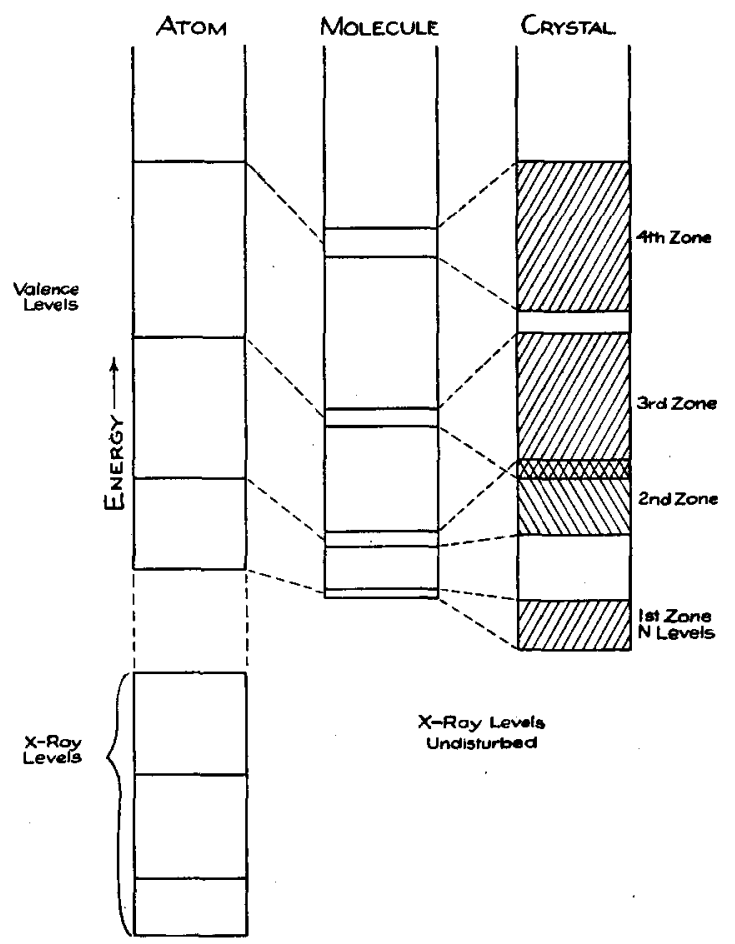

FIG. 1. Hypothetical changes in energy level pattern from atom to molecule to crystal.

The key to all the properties of a solid body is the structure of these zones of energy levels. Let us consider by way of illustration three simple cases.

\section{Case I}

The two lowest bands are widely separated. (Fig. 2(a).) Consider first a crystal composed of atoms having but a single valence electron, e.g., sodium, potassium, silver, etc. Since there are $N$ levels in the lowest band and since two electrons may occupy each level it is evident that when the metal is at the temperature $0^{\circ} \mathrm{K}$ the lower half of the levels will be filled and the upper half entirely vacant. (Fig. 2(b).) There will thus be a sharp upper limit to the energies of the free or valence electrons. But this upper limit of energy turns out to be surprisingly large, of the order of 5 to 10 electron-volts. In the older theories the free electrons in a solid were treated as a perfect gas in which all of the electrons would be at rest and, therefore, have zero energy at $0^{\circ} \mathrm{K}$. Even at $1000^{\circ} \mathrm{K}$ their average kinetic energy would be only about 0.1 electron-volt. The new theory thus predicts that even at $0^{\circ} \mathrm{K}$ 


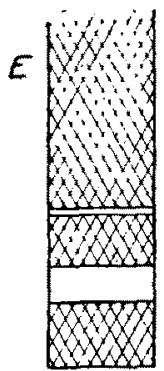

(a)

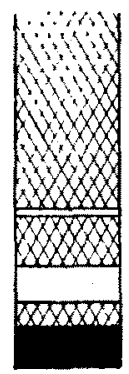

(b)

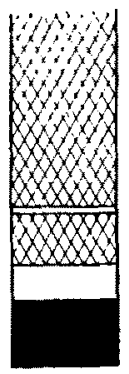

(c)

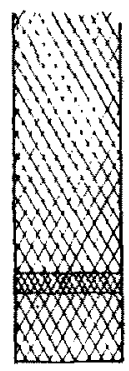

(d)

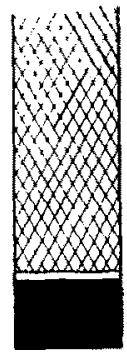

(e)
FIG. 2. Typical zone patterns for crystals. (From Seitz and Johnson.)

the electrons are moving with average kinetic energies 50 to 100 times greater than the classical theories would predict even for high temperatures. This "degenerate" electron gas obeys the Fermi-Dirac statistics rather than the classical Maxwell-Boltzmann statistics.

If now an electric field is applied to such a solid the electrons moving in the direction of the field will attain still higher energies and will move to the higher unoccupied levels in the lowest band. These electrons then move with the field and give rise to an electric current. Electrons in the lower levels cannot do this since the levels directly above are all occupied. Only the relatively few electrons in the upper levels are, therefore, "conducting" electrons. But since, in this case, these electrons do have vacant levels above them to which they can jump, the solid would be a good conductor. All of the monovalent crystals are in fact good electrical conductors; that is, they are metals.

Suppose, however, that we consider a crystal with the same zone pattern but in which there are two valence electrons for each atom. The lower zone will now be completely filled, $2 \mathrm{~N}$ electrons in $N$ levels. (Fig. 2(c).) When an electric field is applied no electron has a vacant state above it into which it can jump. The energy values in the gap between zones are forbidden and an electron could jump over the gap into the allowed levels of the next higher zone only under the influence of an exceedingly strong electric field, for example, $10^{6}$ volts per centimeter. Under ordinary fields, therefore, the electrons cannot acquire additional kinetic energy in the direction of the field. There will, therefore, be no electric current and the solid will be a good insulator. It is thus surprising to find that though this solid has twice as many "free" electrons as the one represented in (b), yet it will be a good insulator while the first will be a good conductor. This is the first time that any theory has given a picture of the fundamental reason why certain crystals are insulators while others are conductors. The necessary condition for conduction is that there shall be unfilled but allowed levels immediately adjacent to the occupied levels.

\section{Case II}

The first two zones overlap. (Fig. 2(d).) There are many crystals which fall into this class. In this case evidently the crystal will be a good conductor even though there are two or three valence electrons per atom since in any case there will be unoccupied levels available. The di- and trivalence metals belong to this class.

\section{Case III}

The gap between the first two zones is very small. (Fig. 2(e).) In this case at the higher temperatures a few electrons will be able to jump the small gap into the unoccupied levels of the second zone even though the first zone is completely filled. These electrons will be able to take part in conduction. In this case the crystal will be a semi-conductor and will show a very strong temperature dependence of its resistance. Transitions to the upper zone may also be caused by light of suitable frequency and photoconduction will result.

We thus see that the simple electrical properties of crystals may be interpreted in terms of their zone structure. By a similar argument the thermal conductivity of crystals may be predicted and it is evident that the condition for electrical conductivity, namely, the existence of available unoccupied levels, is also the condition for thermal conductivity. For only then can the electrons gain energy when the crystal is heated, and carry this energy to the cooler portions. Hence a crystal with good electrical conductivity will also have good thermal conductivity, in agreement with experience. In fact the empirical Wiedemann-Franz law states that the ratio of thermal to electrical conductivities is approximately constant. The new theory calculates a 
value for this constant which is in close agreement with the observed values.

In a similar way the theory can be extended to predict the mechanical properties of the crystal such as the binding energy. This is largely determined by the difference in the total energy of all the electrons in the isolated atoms as compared to the total energy of the electrons in the crystal. If the energy level pattern is known, the energy difference can be computed.

The optical absorption of the crystal also depends upon the zone pattern. The crystal will absorb those light frequencies which will cause the electrons to undergo transitions from an occupied level to an allowed unoccupied level. (In this case conservation of momentum must be observed, and this greatly restricts the allowable transitions.) The electron thus absorbs energy from the light wave. If the light frequency is not such as to cause an allowed transition the light will not be absorbed and the crystal will be transparent to such frequencies.

There are other solid properties which the new theory has successfully handled. The mechanisms of photoelectric and thermionic emission are better understood than ever before. Measurements of the energy distribution of emitted photoelectrons has provided direct experimental evidence for the reality of the Fermi-Dirac energy distribution of the electrons in the metal. The magnetic properties of crystals have also been treated and for the first time a satisfactory theory of ferromagnetism is being developed.

These remarks may serve to convey in a crude and incomplete way some aspects of the general picture on which the new theory of solids is being built. ${ }^{1}$ Only a few years ago the theory of

1 These developments are more fully discussed with a bibliography in the series of articles by Seitz and Johnson solids was in a chaotic state; it consisted in fact of many contradictory theories, each designed to explain particular phenomena and having no relation to the others. In the light of this fact the new theory must be regarded as startlingly successful since it provides a single underlying picture which correlates all solid properties. The two essential features of this picture are (1) the crystal is treated as a single molecule with definite energy levels and (2) the motion of the electron in the crystal is treated as a wave motion. Actually (1) is a necessary consequence of (2).

I do not wish to imply, however, that the field is all finished. On the contrary its development has only begun. The groundwork seems to be firmly laid but there are many aspects which are offering difficulties (particularly the so-called structure sensitive properties of crystals which depend greatly on slight irregularities, traces of impurities, etc.). Superconductivity is only imperfectly understood. Other features of solids have not been explained because of mathematical difficulties. In many of these cases, however, even though the theory does not provide quantitative treatment, its qualitative ideas have proved of value and a stimulus to further experimental work. Fluorescence, photoconductivity and photographic effects are examples.

Thus we see that even so abstract a thing as quantum mechanics, arising from the abstract field of atomic structure, may come in to "practical" use and may even affect workshop procedure. Electron waves may seem to be intangible things, but they give rise to important results. This constitutes a fine example of the "practical" consequences of "impractical" science.

(J. App. Phys. 8, 84, 186, 246 (1937)). The remarks in the present paper are largely extracts from these articles. 